\title{
Energy Efficiency of Very Large Multiuser MIMO Systems with Transmit Antenna Selection
}

\author{
Jie Li, Shuangzhi Li, Xiaomin Mu and Jiankang Zhang \\ School of Information Engineering, Zhengzhou University, Zhengzhou, China \\ E-mail:ielijie@sina.com, shuangzhilee@gmail.com,iexmmu@zzu.edu.cn, \\ iejkzhang@zzu.edu.cn
}

\begin{abstract}
In this paper, transmit antenna selection is performed to improve the energy efficiency of multiuser large scale MIMO systems, which is necessary for the energy efficiency maximization of large scale MIMO, because the RF chains which are connected with antennas are very expensive and consume a lot of power. However, the consumed power of the large scale MIMO systems should include not only transmit power but also the fundamental power for operating the circuit at the transmitter, because the effect of circuit power consumption is more serious when the transmitter is equipped with massive number of antennas. We evaluate the performance of the energy efficiency in the multiuser case where zero-forcing precoding is used to reduce the inter-user interference. The theoretical analysis indicates that there exists an optimal number of selected antennas to maximize the energy efficiency, and we derive optimal antenna number for different number of users. All the analytical results are verified through both mathematical analysis and numerical simulations.
\end{abstract}

Keywords: energy efficiency, transmit antenna selection, multiuser MIMO system, large scale MIMO system

\section{Introduction}

The problem of energy efficiency has mainly been studied for power-limited applications [1] in the past years. However, with growing energy demands and increasing energy price, this problem has been noticed in the development of future mobile cellular systems [2]. Recently, there has been a great deal of interest in large scale multiuser MIMO systems, which typically means that wireless systems with hundreds of antennas serve tens of users simultaneously. Simple signal detectors are proposed to alleviate the computational complexity problem in multiple antenna systems, and [3] shows that it can reduce the transmit power of each user proportionally to $1 / \mathrm{M}$ if the Base Station (BS) has perfect channel state information (CSI) when the number of BS antennas M grows without bound. However, one of the disadvantages of employing multiple antennas is the associated complexity which results from employing a separate radio frequency (RF) chain for every employed antenna, which will greatly limit the practical application of large MIMO technology.

The antenna selection scheme is proposed to solve the aforementioned problems, which has been intensively studied in previous literatures [4-6]. The theoretical derivation has been carried on in [4] to prove that the capacity of MIMO systems with antenna selection scheme is better than the systems without selection scheme. An optimal antenna selection algorithm of non-full rank channel has been addressed in [5], which needs to go through all the possible antenna subset with high computational complexity. The scenario when the antenna selection algorithms are applied to the receiving end in single-user (SU) MIMO system is also studied in the 
aforementioned literatures. [6] proposes an antenna selection algorithm for receiving end in multiuser MIMO system, which improves the capacity of downlink. Two antenna selection schemes maximizing the energy efficiency of the single-user massive MIMO systems are proposed in [11], without the consideration of the problem of energy efficiency maximization in the multiuser scenario. To the best of our knowledge, however, there are very few previous papers that study the downlink performance with transmitting antenna selection in multiuser large scale MIMO system.

In this paper, we perform transmit antenna selection to improve the energy efficiency of multiuser large scale MIMO systems. Under such a scenario, we establish a new power consumption model as the addition of the transmit power and the circuit power consumption, and based on this model, analyze the effects of the number of transmitting antennas at the base station and the number of users at the receiver on the total power consumption and the energy efficiency respectively. We focus on the multiuser scenario where the zero-forcing (ZF) precoding is used to reduce inter-user interference, and we analytically derive the optimal number of selected antennas which can maximize the energy efficiency in this case. Based on the optimal number of transmit antenna, we compare the energy efficiency improvement by antenna selection with using all of the given transmit antennas.

\section{System Model}

We consider the downlink of a large scale multiuser MIMO system with one BS and $K$ terminals, as shown in Figure 1. The BS is equipped with $N_{t}$ transmit antennas, and each terminal has only one receive antenna, which is common for a cellphone. We assume that $N_{t}$ is large and $N_{t} \square K$.

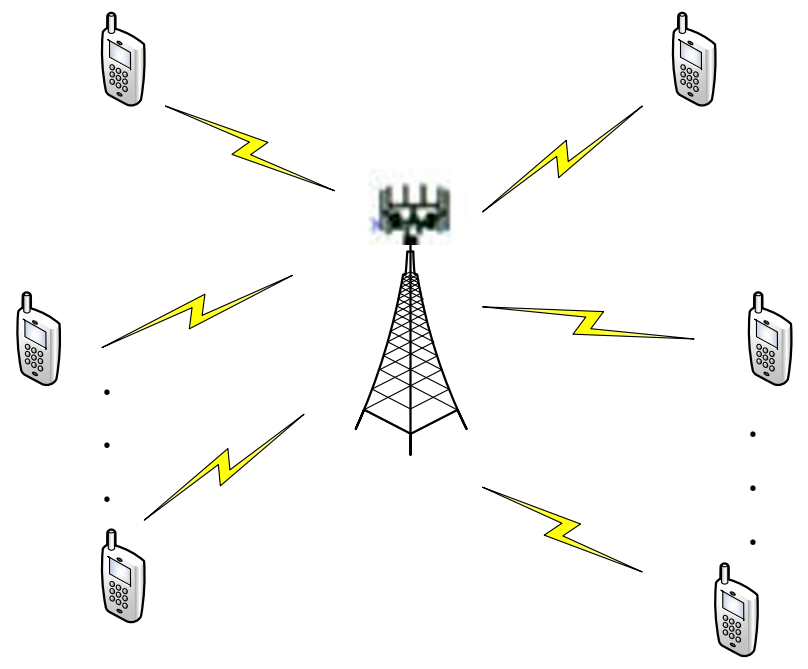

Figure 1. The Large Scale Multiuser MIMO System Model

It is impractical to use all the antennas to achieve high rate due to the expensive RF chains and high hardware complexity. Thus, we perform transmit antenna selection to choose the best $N^{*}$ antennas from all the available $N_{t}$ ones. We assume perfect CSI at the transmitter.

For multiuser MIMO, the signal for a user is interference for the others and it is generally impossible to cooperate among receiver sides in the downlink of multiuser MIMO, so the achievable rate of each user is limited by multi-user interference 
(MUI) and noise. So we consider the zero-forcing (ZF) precoding scheme to reduce MUI. The receive signal vector at terminals can be written as

$$
\mathbf{y}=\sqrt{\frac{P_{t x} N_{t}}{K}} \mathbf{H W} \mathbf{x}+\mathbf{n}
$$

where $\mathbf{y}$ is the $K \times 1$ received vector for each terminal, $\mathbf{x}$ is the transmitted signal vector, $P_{t x}$ is the total transmit power, $\mathbf{H} \in \square^{K \times N_{t}}$ is the channel matrix between $N_{t}$ BS antennas and $K$ terminals, $\mathbf{n}$ is $K \times 1$ noise (AWGN) vector. $\mathbf{W}$ is the $\mathrm{ZF}$ precoding matrix and $\mathbf{W}=\mathbf{H}^{H}\left(\mathbf{H H}^{H}\right)^{-\mathbf{1}}$. Then the receive signal of $k$-th user is

$$
y_{k}=\sqrt{\frac{P_{t x} N_{t}}{K}} \mathbf{H}_{k} \mathbf{W}_{k} x_{k}+\sqrt{\frac{P_{t x} N_{t}}{K}} \sum_{i=1, j \neq k}^{K} \mathbf{H}_{k} \mathbf{W}_{i} x_{i}+n_{k}
$$

where the second term on the right side is MUI, $\mathbf{H}_{k}, \mathbf{W}_{k}, x_{k}$ are the channel matrix, precoding matrix and transmitted signal corresponding to user $k$, respectively. The achievable sum rate can be represented as

$$
C=\lambda B \cdot \sum_{k=1}^{K} E\left[\log _{2}\left(1+\frac{\frac{P_{t x} N_{t}}{K}\left|\mathbf{H}_{k} \mathbf{W}_{k}\right|^{2}}{\frac{P_{t x} N_{t}}{K}\left|\sum_{i=1, i \neq k}^{K} \mathbf{H}_{k} \mathbf{W}_{i} x_{i}\right|^{2}+N_{0} B}\right)\right]
$$

where $\lambda$ is the scaling factor for pilot overhead and guard interval, and $N_{0} B$ is the noise power in the given bandwidth $B$. In the multiuser large scale MIMO system, (3) can be simplified as

$$
C_{\text {approx }} \approx \lambda B K \cdot \log _{2}\left(1+\frac{P_{t x} N_{t}}{\left(I+N_{0} B\right) \mathrm{K}}\right)
$$

where $I$ is the MUI term, which can be disregarded in the case of ZF precoding.

\section{Analysis of Energy Efficiency with Transmit Antenna Selection}

In this paper, we define the energy efficiency as

$$
E E=\frac{C}{P_{\text {total }}}
$$

$P_{\text {total }}$ is the total power consumption which includes both the transmit power $P_{t x}$ and the circuit power consumption $P_{c i r}$ in our model, i.e., $P_{t o t a l}=P_{t x} / \eta+P_{c i r} . \eta$ is the power amplifier efficiency. In this paper, the circuit power consumption is modeled as

$$
P_{\text {cir }}=P_{B}+P_{s y n}+N_{t}\left(P_{D A C}+P_{m i x}+P_{\text {filt }}\right)
$$

where $P_{B}$ is the baseband power consumption, and $P_{s y n}, P_{D A C}, P_{m i x}, P_{f i l t}$ are the power consumption values for the frequency synthesizer, the DAC, the mixer, the filters at the transmitter side, respectively.

For multiuser MIMO, we consider the $10 \mathrm{MHz}$ OFDM system. And the large scale multiuser MIMO baseband computation model which was presented in [7] is used

$$
P_{B}=\frac{\varphi(\text { Gflops })}{\vartheta(\text { Gflops } / W)}
$$

where $\vartheta$ is the very large scale integration processing efficiency. The $\varphi($ Gflops $)$ can be expressed as 


$$
\begin{aligned}
\varphi= & N_{t} B \cdot\left[\frac{T_{u}}{T_{s}} \cdot\left(1-\frac{T_{p}}{T_{s l}}\right) \mathrm{K}+\frac{T_{p}}{T_{s l}} \mathrm{~K}^{2}\right. \\
& \left.+\frac{T_{u}}{T_{s}} \cdot \log _{2}\left(T_{u} B\right)+\frac{T_{u}}{T_{s}} \cdot \frac{T_{p}}{T_{s l}} \log _{2}\left(\frac{T_{u}}{T_{s}} \cdot \frac{T_{p}}{T_{d}}\right)\right]
\end{aligned}
$$

Based on the achievable sum rate (4) and the above total power consumption model, the energy efficiency in (5) can be formulated as

$$
E E=\frac{C}{P_{\text {total }}} \approx \frac{\lambda B K \cdot \log _{2}\left(1+\frac{P_{t x} N_{t}}{\left(I+N_{0} B\right) \mathrm{K}}\right)}{P_{t x} / \eta+P_{\text {cir }}}
$$

There is a tradeoff between the power consumption and the achievable sum rate. The energy efficiency increases as increasing the number of antennas, while the power consumption increases at the same time, resulting in concave shape of energy efficiency curve. In this paper, we investigate an appropriate number of selected transmit antennas $N^{*}$ for energy efficiency maximization, and the optimization problem can be defined as

$$
N^{*}=\arg \max _{N_{t}} \frac{C}{P_{\text {total }}}
$$

As the energy efficiency function is concave, then, we can derive the optimal number of selected transmit antennas, $N^{*}$, which should satisfy the following equation

$$
\begin{aligned}
\frac{\partial(E E)}{\partial N_{t}} & \approx \frac{\partial}{\partial N_{t}}\left(\frac{C_{\text {approx }}}{P_{\text {total }}}\right) \\
& =\frac{P_{t x} B K}{N_{0} B \mathrm{~K} P_{\text {total }} \log _{2} e \cdot\left(1+\frac{P_{t x} N_{t}}{N_{0} B \mathrm{~K}}\right)}-\frac{B K P \cdot \log _{2}\left(1+\frac{P_{t x} N_{t}}{N_{0} B \mathrm{~K}}\right)}{P_{\text {total }}^{2} \log _{2} e} \\
& =0
\end{aligned}
$$

Then, $N^{*}$ can be written as

$$
N^{*} \approx \frac{N_{0} B \mathrm{~K}}{P_{t x}}\left(\mathrm{e}^{1+\mathrm{W}(\Gamma)}-1\right)
$$

where

$$
\Gamma=\frac{N_{t} P_{t x}^{2} / \eta-N_{0} B K P_{c i r}}{N_{0} B \mathrm{~K} P_{c i r} \cdot \mathrm{e}}
$$

and $W$ is the lambert $W$ function, we can easily get the optimal selected antenna number $N^{*}$ from (12). And now the problem is how to select the $N^{*}$ antennas from the total transmit antennas.

When $N^{*}$ is fixed, $P_{\text {total }}$ is independent of antenna selection, so the antenna selection for energy efficiency equals the antenna selection for channel capacity. For the optimal antenna selection, we need to choose $N^{*}$ columns of $\mathbf{H}$ to maximize the energy efficiency,

$$
\mathbf{H}_{\text {sel }}=\arg \max _{\left\{\mathbf{H}_{\text {sel }} \in \mathbf{H}\right\}} \frac{C\left(\mathbf{H}_{\text {sel }}\right)}{P_{\text {total }}}
$$


where $\left\{\mathbf{H}_{\text {sel }} \in \mathbf{H}\right\}$ is the set of all possible combination of antennas. Note that optimal antenna selection algorithm has a complexity of $\left(\begin{array}{l}N_{t} \\ N^{*}\end{array}\right)$, which means the complexity is extremely high in large scale MIMO system.

According to the results in [8], for large $N_{t}$ and fixed $K$, the numerically accurate approximation of the distribution of the mutual information with full complexity is given by

$$
I \sim \mathcal{N}\left(K \cdot \log _{2}\left(1+N_{t} P_{t x}\right), \frac{K \cdot\left(\log _{2} e\right)^{2}}{N_{t}}\right)
$$

As can be seen from (15), for fixed $K$, when $N_{t} \rightarrow \infty$, the channel quickly "hardens", in the sense that the mutual information fluctuation decreases rapidly relative to its mean, which means that if we use numerous transmit antennas with limited users, the channel condition for each user is nearly constant. Based on this conclusion, the channel hardening phenomenon [8] provides the large scale multiuser MIMO with the advantage of making all users' channel condition almost evenly good. Thus random antenna selection is nearly optimum with lower complexity. Therefore, if the number of selected antennas $N^{*}$ is determined,

$$
C_{\text {sel }}^{\text {rand }} \approx \lambda B K \cdot \log _{2}\left(1+\frac{P_{t x} N^{*}}{N_{0} B K}\right)
$$

\section{Simulation Results}

In this section, we evaluate the energy efficiency for the large scale multiuser MIMO system with transmit antenna selection. The simulation parameters are shown in Table 1 and the value of parameters follows the setting of [9-10].

\section{Table 1. Performance Evaluation Parameters}

\begin{tabular}{|c|c|}
\hline Parameters & Settings \\
\hline$P_{s y n}, P_{D A C}, P_{m i x}, P_{f i l t}(m W)$ & $50,15.6,30.3,20$ \\
\hline$\eta$ & 0.38 \\
\hline$B(\mathrm{MHz})$ & 10 \\
\hline$\vartheta(G f l o p s / \mathrm{W})$ & 50 \\
\hline$T_{s l}, T_{p}(m s)$ & $0.5,0.214$ \\
\hline$T_{s}, T_{u}, T_{g}, T_{d}(u s)$ & $71.4,66.7,4.7,4.7$ \\
\hline
\end{tabular}

The effect of the number of transmit antennas on the variance of the mutual information based on (15) is plotted in Figure 2, and we can find that in a large scale multiuser MIMO system with fixed $K$, as the number of antennas grows, the variance approaches to zero. So we can conclude that the channel hardening phenomenon exists in the large scale multiuser MIMO systems, providing the system with the advantage of making the channel condition for each user is nearly constant. That's why we use random antenna selection instead of optimal antenna selection for lower complexity in this paper. 


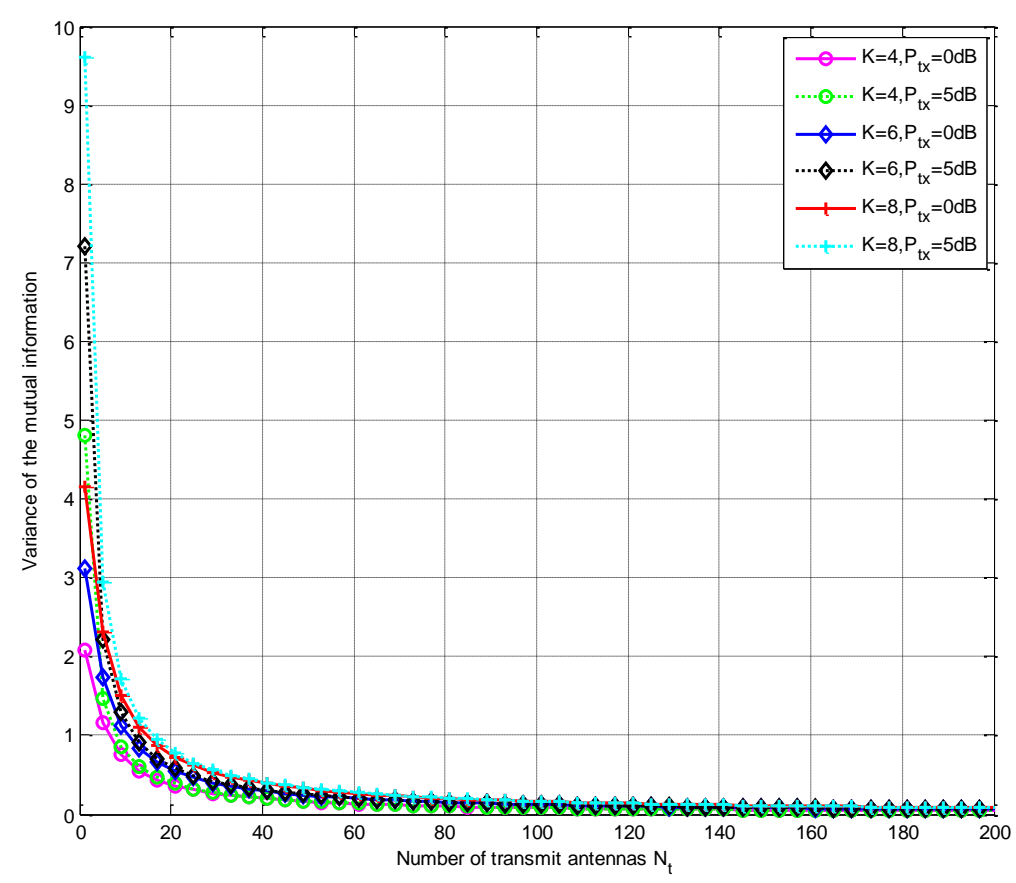

\section{Figure 2. The Effect of the Number of Transmit Antennas on the Variance of the Mutual Information}

Based on the new power consumption model, the effects of the increase in the transmit antennas and the users on the tendency of increase in total power consumption are shown in Figure 3 and Figure 4, respectively. Since the circuit power consumption from analog devices is considered, the total power consumption is gradually increasing. In the aspect of energy efficiency, the increase in the total power consumption affects the tendency of energy efficiency critically.

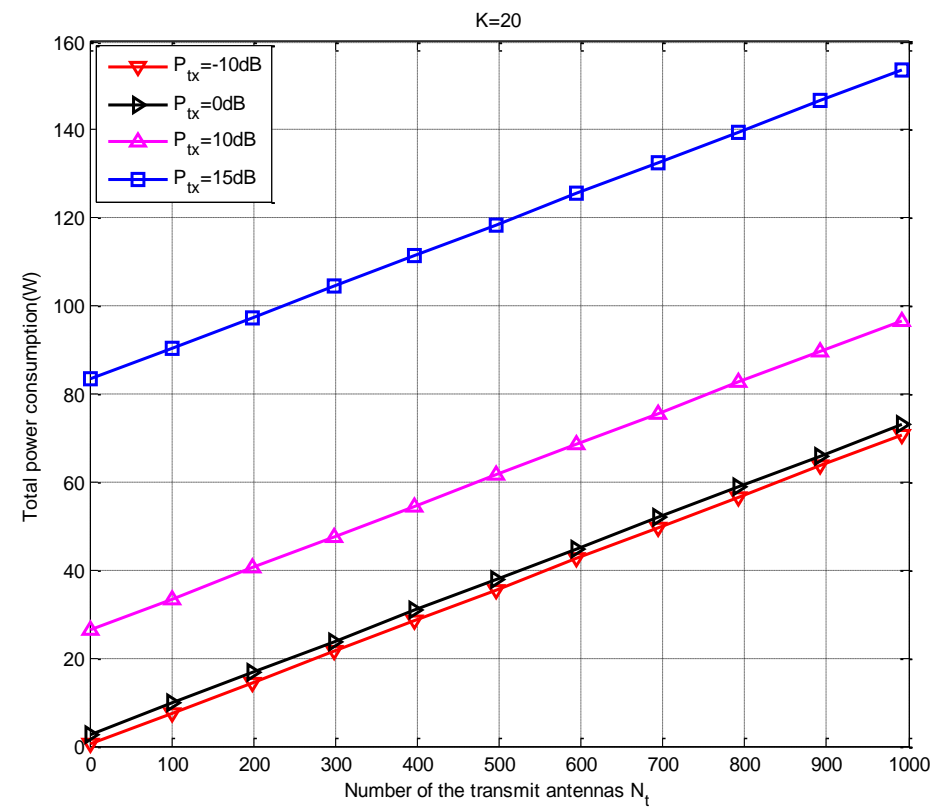

Figure 3. Total Power Consumption Versus the Number of Transmit Antennas 


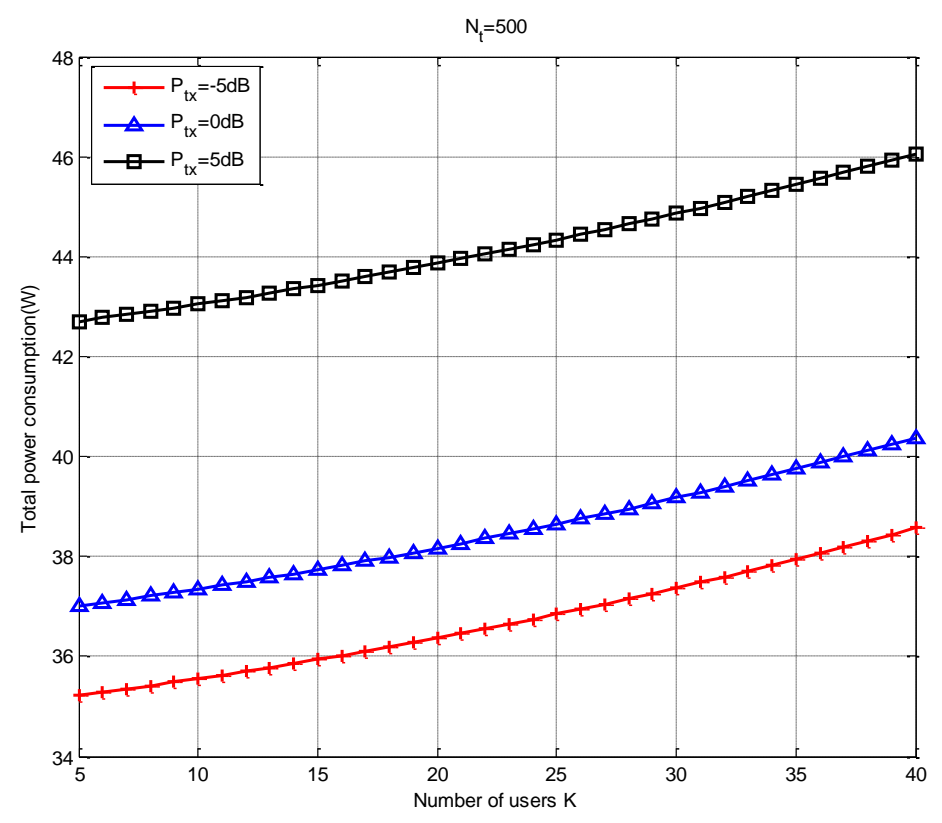

Figure 4. Total Power Consumption Versus the Number of Users

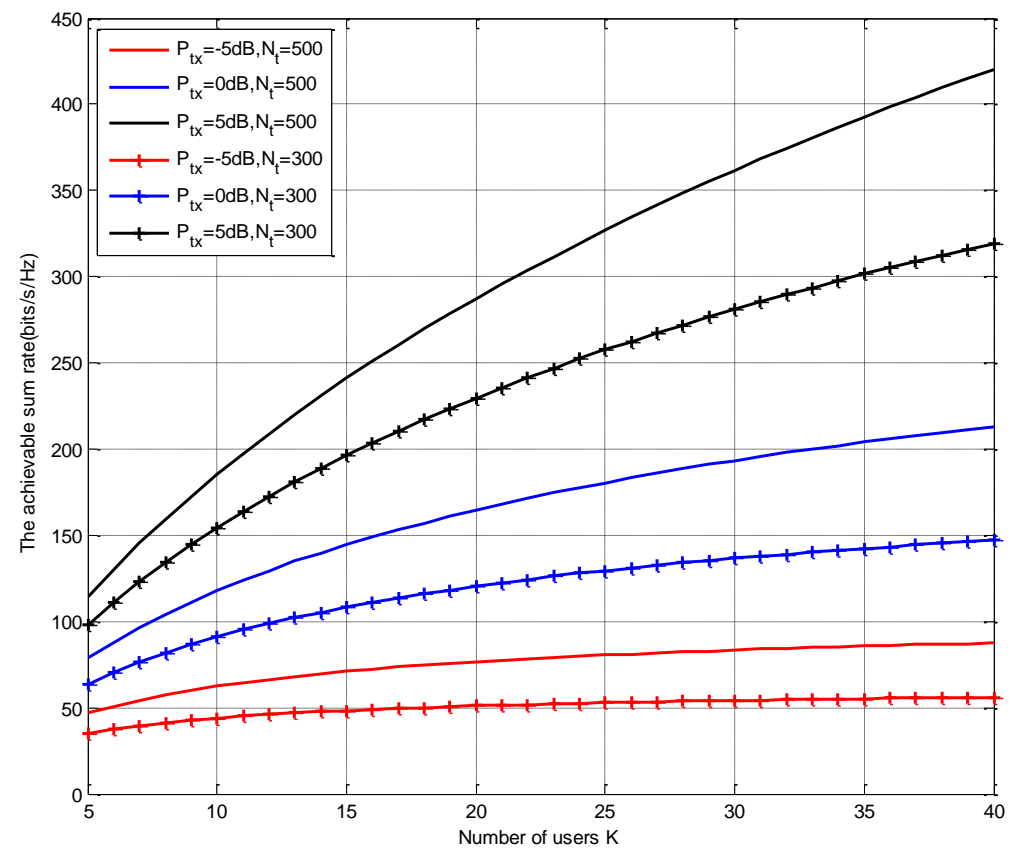

Figure 5. The Achievable Sum Rate Versus the Number of Users with Different $P_{t x}$ and $N_{t}$ 


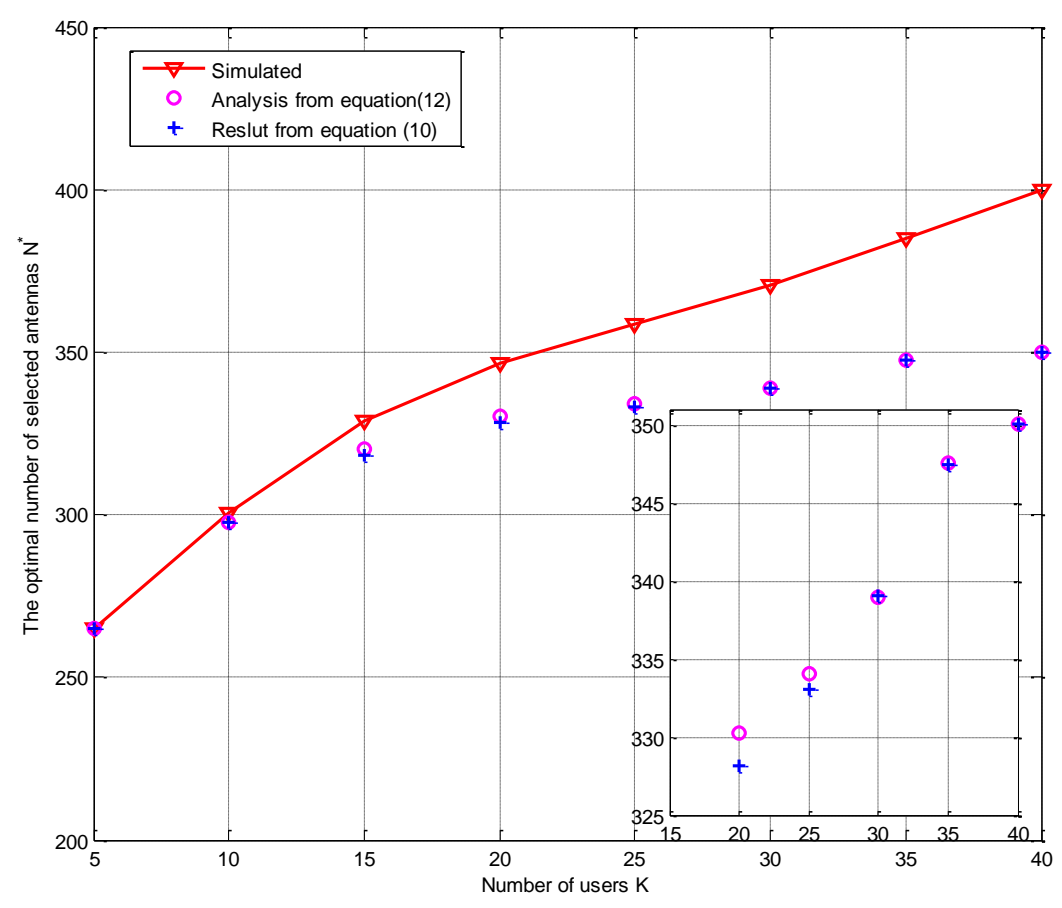

Figure 6. The Optimal Selected Antennas Number Versus the Number of Users

The achievable sum rate for different numbers of users under different BS transmit antennas and different transmit powers for ZF with perfect CSI is shown in Figure 5. According to the results from Figure 3, Figure 4 and Figure 5, we can conclude that in multiuser large scale MIMO systems, there is a tradeoff between the total power consumption and the achievable sum rate. The achievable sum rate increases as increasing the number of users and transmit antennas while the total power consumption also increases, which results in the existence of the optimal number of selected antennas to maximize the energy efficiency when $K$ is fixed.

The comparison between the simulation and our derived results for optimal antenna number $N^{*}$ with regard to the number of users $K$ is shown in Figure 6, when $N_{t}=1000, P_{t x}=40 \mathrm{~W}$. The purple 'o' stands for the analysis from (12) and the blue ' + ' stands for the result from maximizing the energy efficiency (10) based on the equation (9), when ZF precoding is used. As the equation (12) and (9) are obtained by approximation, when the number of transmit antennas is fixed, the discrepancy between the simulated and theoretical results is only related to $K$. The larger $K$ is, the larger the discrepancy is. As shown in Figure 6, it's obvious that the theoretical analysis matches the simulation better when the gap between $N_{t}$ and $K$ is larger. 


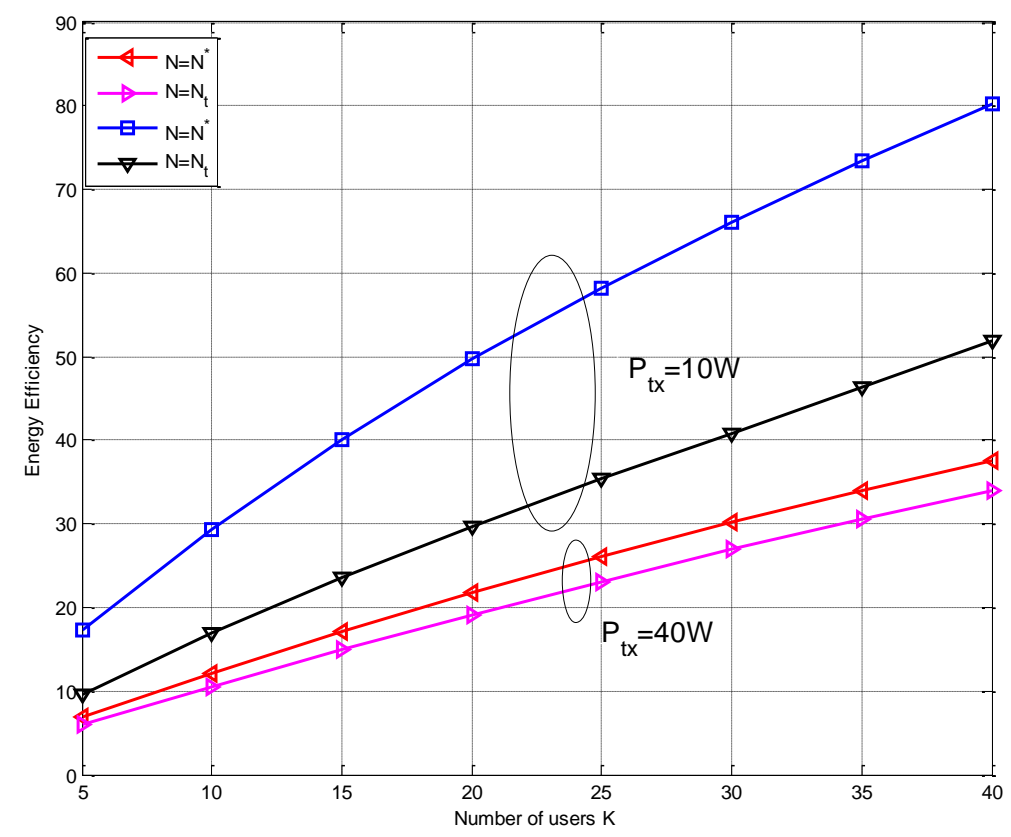

Figure 7. Energy Efficiency Improvement through Transmit Antenna Selection

Figure 7 indicates the energy efficiency performance improvement by transmit antenna selection under different transmit powers. Especially, when the number of users is 10 , and the transmitting power is $40 \mathrm{~W}$ and $10 \mathrm{~W}$, compared with using all of the given transmitting antennas, the proposed antenna selection scheme achieves nearly $12 \%$ and $78 \%$ gain in energy efficiency, respectively. Simulation results indicate that transmit antenna selection in large scale multiuser MIMO system can improve the energy efficiency significantly.

\section{Conclusions}

In this paper, we accessed the energy efficiency performance of the large scale multiuser MIMO systems with transmit antenna selection. Through the mathematical derivation and simulation results, we obtained that when the transmit power and the circuit power consumption are both considered, there exists an optimum number of selected antennas to maximize the energy efficiency under ZF precoding with perfect CSI. And the simulation results also showed that transmit antenna selection in large scale multiuser MIMO systems can improve energy efficiency significantly comparing with using all of the transmit antennas, which is proved to be a candidate technique for green communications in future wireless standards.

\section{Acknowledgements}

The financial support of the National Natural Science Foundation of China under grants (No. 61271421 and No. 61301150), and the Specialized Research Fund for the Doctoral Program of Higher Education (SRFDP) under grants (No. 20134101120001) are gratefully acknowledged. 


\section{References}

[1] K. Lahiri, A. Raghunathan, S. Dey and D. Panigrahi, "Battery-driven system design: A new frontier in low power design," Proceedings of the 15th International Conference on VLSI Design, Bangalore: IEEE, (2002), pp. 261-267.

[2] L. Song and J. Shen, "Evolved cellular network planning and optimization for UMTS and LTE," CRC Press, Florida, (2010).

[3] H. Q. Ngo, E. G. Larsson and T. L. Marzetta, "Energy and spectral efficiency of very large multiuser MIMO systems," IEEE Transactions on Communications, vol. 61 no. 4, (2013), pp. 1436-1449.

[4] A. F. Molisch, M. Z. Win, Y. Choi and J. H. Winters, "Capacity of MIMO systems with antenna selection," IEEE Transactions on Wireless Communications, vol. 4 no. 4, (2005), pp. 1759-1772.

[5] D. A. Gore, R. U. Nabar, and A. Paulraj, "Selecting an optimal set of transmit antennas for a low rank matrix channel," Proceedings of IEEE International Conference on Acoustics, Speech, and Signal Processing, Istanbul: IEEE, (2000), pp. 2785-2788.

[6] Y. Wu, J. Zhang, H. Zheng, X. Xu and S. Zhou, "Receive antenna selection in the downlink of multiuser MIMO systems," IEEE 62nd Vehicular Technology Conference, (2005), pp. 477-481.

[7] H. Yang and T. L. Marzetta, "Total energy efficiency of cellular large scale antenna system multiple access mobile networks," IEEE Online Conference on Green Communications, Piscataway, New Jersey: IEEE, (2013), pp. 27-32.

[8] B. M. Hochwald, T. L. Marzetta and V. Tarokh, "Multiple-Antenna Channel Hardening and Its Implications for Rate Feedback and Scheduling," IEEE Transactions on Information Theory, vol. 50 no. 9, (2004), pp. 1893-1909.

[9] D. Ha, K. Lee and J. Kang, "Energy Efficiency Analysis with Circuit Power Consumption in Massive MIMO Systems," IEEE 24th International Symposium on Personal Indoor and Mobile Radio Communications, London, United Kingdom: IEEE, (2013), pp. 938-942.

[10] T. L. Marzetta, "Noncooperative Cellular Wireless with Unlimited Numbers of Base Station Antennas," IEEE Transactions on Wireless Communications, vol. 9 no. 11, (2010), pp. 3590-3600.

[11] H. Li, L. Y. Song and M. Debbah, "Energy Efficiency of Large-Scale Multiple Antenna Systems with Transmit Antenna Selection," IEEE Transactions on Communications, vol. 62 no. 2, (2014), pp. 638647.

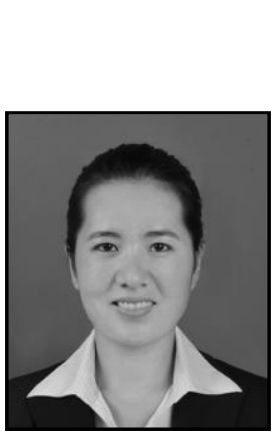

\section{Authors}

Jie Li, she received her B. S. degree in Electronic and Information Engineering from Zhengzhou University in 2012. She is now a graduate student at the Zhengzhou University. Her main research interests are in the areas of antenna selection technique in MIMO systems.

Shuangzhi Li, he received his B. S. degree from Zhengzhou University in 2012. He is now a graduate student at the Zhengzhou University. His research concerns channel estimation for multiuser OFDM / SDMA system.

Xiaomin Mu, she received her B. S. degree from Beijing Institute of Technology in 1982. She is now a professor and also the $\mathrm{PhD}$ supervisor. Her research concerns multiantenna wireless communication systems, communication information processing, cognitive radio, image signal processing, etc.

Jiankang Zhang, he received his PhD degree from Zhengzhou University in 2012. He is now an associate professor. His research concerns multi-antenna wireless communication system, communication information processing, etc. 